\title{
Validity and reproducibility of the FFQ (FFQW82) for dietary assessment in female adolescents
}

\author{
Mariko Watanabe ${ }^{1}$, Kazue Yamaoka ${ }^{2, *}$, Masako Yokotsuka ${ }^{1}$, Misa Adachi ${ }^{3}$ and \\ Toshiro Tango ${ }^{2}$ \\ 'Department of Human Nutrition, Graduate School of Human Ecology, Showa Women's University, Tokyo, \\ Japan: ${ }^{2}$ Department of Technology Assessment and Biostatistics, National Institute of Public Health, 2-3-6 \\ Minami, Wako, Saitama 351-0197, Japan: ${ }^{3}$ Doctoral Course of National Institute of Public Health, \\ Saitama, Japan
}

Submitted 5 November 2008: Accepted 23 April 2010: First published online 11 June 2010

\begin{abstract}
Objective: To assess the validity and reproducibility of a self-administered FFQ with eighty-two food items (FFQW82) for assessing the habitual diet in female adolescents.

Design: The validity of the FFQW82 for assessment of nutrient intake was evaluated by comparison with a $7 \mathrm{~d}$ weighed food record (7d-FRRI) reported as 'gold standard'. Reproducibility of the FFQW82 was assessed at an interval of 1 month (test-retest method). The first survey (FFQW82 and 7d-FRRI) was conducted in April 2007 and the second FFQW82 survey was conducted in May 2007. Daily consumption of energy from eleven food groups and nine nutrients were calculated from both instruments for breakfast, lunch, dinner and the whole day. Crude and energyadjusted Pearson correlation coefficients were calculated using log-transformed data. Setting: Middle school, Tokyo, Japan.

Subjects: Female adolescents aged 12-13 years.

Results: Sixty-three female adolescents completed both surveys. The relative difference between the energy intake calculated by the FFQW82 and the 7d-FRRI for the whole day, breakfast, lunch and dinner was $8 \%, 10 \%, 15 \%$ and $10 \%$, respectively. As for validity, the correlation coefficient of total energy intake for the whole day was $0 \cdot 31$. The result for breakfast was relatively higher $(0.59)$ compared with that for lunch $(0 \cdot 40)$ and dinner $(0 \cdot 32)$. For macronutrients, the energy-adjusted correlation coefficient ranged from $0 \cdot 28$ (carbohydrates) to 0.53 (protein). Reproducibility of total energy intake was 0.62 and ranged from 0.46 (fat) to 0.69 (carbohydrate) for macronutrients.

Conclusions: These results suggest that the FFQW82 has proved to have some potential with regard to reproducibility among our study population.
\end{abstract}

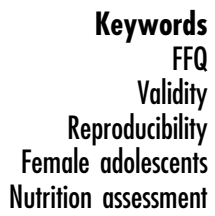

FFQ

Validity

Female adolescents Nutrition assessment
In recent years, youth obesity ${ }^{(1,2)}$ and lifestyle-related diseases $^{(3-5)}$ have increased significantly, as have problems related to a distorted body image ${ }^{(6)}$. The accurate assessment of nutritional intake is important in epidemiological studies. Although under-reporting of energy intake in young people has been shown ${ }^{(7)}$, the FFQ is feasible for this purpose ${ }^{(8)}$, considering the circumstances of young people that prohibit them from weighing foods, such as school attendance, as well as their ability to meticulously record food intake.

Thus far, various FFQ have been assessed to determine their validity for use in young people in Western countries $^{(9-16)}$. To our knowledge, however, no study has determined the effectiveness of an FFQ in acquiring information on the diets of young people in Japan. Previously, we developed an FFQ with sixty-five food items (FFQW65) to be used in nutritional education for the prevention of diabetes $^{(17)}$. This instrument assessed energy intake separately for breakfast, lunch and dinner in addition to intake for the entire day and was useful for nutritional education in adults. We reported on the assessment of the effect of nutritional education in a randomized controlled trial for a high-risk group of persons with diabetes using the FFQW65 ${ }^{(17)}$. In the last decade, however, dietary habits and the variety of foods eaten by the Japanese have changed. To address the diversity of the current diet, we have newly developed an FFQ with eighty-two food items (FFQW82). The validity and reproducibility of the FFQW82 in adults was evaluated elsewhere ${ }^{(18)}$. To adapt the FFQW82 for use in adolescents, it must be evaluated using young subjects. Our earlier work $^{(19)}$ in adults looked at eating patterns for each meal among persons with diabetes, and the 
assessment led to success in nutrition education to correct excess eating at night and to increase dietary intake at breakfast. The younger generation in Japan tends to eat little for breakfast, and correcting that eating pattern is an objective of our nutrition education. Thus, it is important to ascertain the breakfast habits of this age group. The FFQW82 may be a useful instrument for this purpose. Thus the aim of the present study was to evaluate the relative validity and reproducibility of the FFQW82 for measuring dietary intake in female adolescents.

\section{Methods}

\section{Subjects and study design}

Subjects were 121 female adolescents aged 12-13 years who attended a private junior high school in Tokyo. The FFQW82 was validated by comparing data from that instrument with data obtained by an alternative dietary assessment method, a $7 \mathrm{~d}$ weighed food record reported intake (7d-FRRI). Of the 121 subjects, sixty-three completed the 7d-FRRI for at least six of the seven days $(52 \cdot 1 \%$ of study subjects). Because many of the students usually bought their lunch while at school, a recall method was used for weekday lunch while the full procedure for the 7d-FRRI was used for all other meals, snacks, etc. An electronic scale was loaned to each subject to quantify the food portions consumed. Subjects and parents were instructed on how to use household measures and carry out procedures related to the $7 \mathrm{~d}$-FRRI. All students were requested to photograph their meals so that the accuracy of their records could be checked by dietitians. Dietitians attempted to reconstruct missing or ambiguous information after examining the completed 7d-FRRI. The first survey using the FFQW82 (FFQ1) was performed in April 2007. Shortly thereafter, the 7d-FRRI was conducted. Then 1 month later, a second survey using the FFQW82 (FFQ2) was conducted in May 2007 to determine the reproducibility of the FFQW82 (Fig. 1). All responses were reviewed after completion of the survey for possible errors or missed questions.
Reproducibility of the FFQW82 was evaluated using data on sixty of the sixty-three students $(49 \cdot 6 \%$ of study subjects) who were administered both the FFQ1 and FFQ2. Three of the sixty-three students did not fully complete the FFQ2 for various reasons.

The study was approved by the Ethics Committee of Showa Women's University. The purpose of the study and the procedures involved were explained to all study subjects who then provided informed consent.

\section{FFQW82}

The FFQW82 is a self-administered questionnaire on recall of intake frequency and portion size over a period of 1 month from a list of foods with pictures. The FFQW82 can be completed in about $30 \mathrm{~min}$. From responses to the FFQW82, energy and nutrient intake for breakfast, lunch and dinner as well as for the whole day can be estimated. The portion size is described as 'small', 'medium' or 'large'. The standard amount for 'medium' was determined by consulting a past survey (September 2006, for students of the former grade) and was shown by size on the food list. 'Small' is defined as half the amount of 'medium', whereas 'large' is defined as 1.5 times the amount of 'medium'. Intake frequency is indicated by six categories: $(0=$ 'absolutely do not eat'; $1=$ 'eat once or twice per month'; $2=$ 'eat once or twice per week'; $3=$ 'eat 3 to 4 times per week'; $4=$ 'eat 5 to 6 times per week'; and $5=$ 'always eat at breakfast (or lunch or dinner)'). Some of the listed foods comprised more than type of food. For example, the category 'fat' included foods with various ingredients, such as 'stir-fried vegetables', 'deep-fried food', 'stew', 'fried rice', etc. Because some snacks, such as rice balls, can be considered part of a meal, it was not always possible to specify what was a snack or a meal. In these cases, we treated some snacks as meals. The estimated intake of energy and several nutrients for a whole day was calculated by simply summing up the estimated intake for each meal. Subjects were instructed to recall and then record what they ate and drank, including betweenmeal snacks or a food eaten multiple times per day. The Microsoft ${ }^{\circledR}$ Excel 2007 program (Microsoft Inc., Redmond,

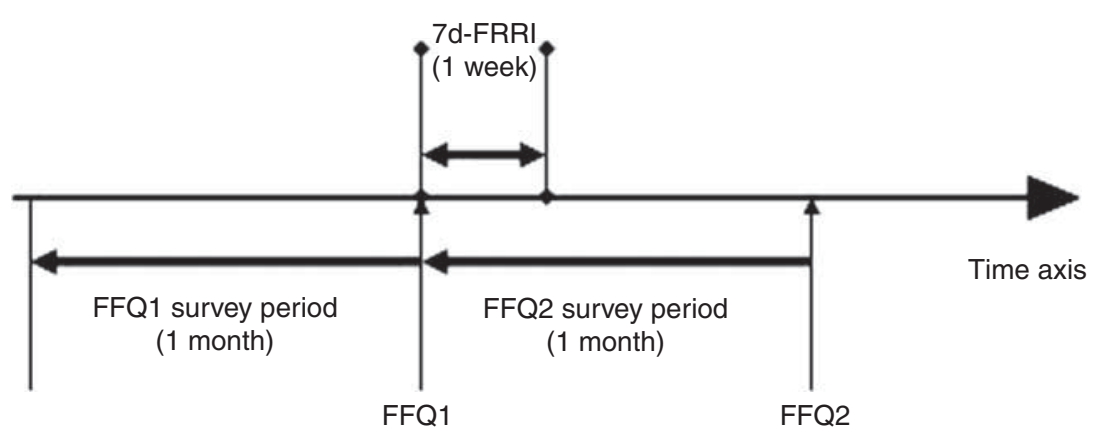

Fig. 1 Process of assessment using FFQW82 and 7d-FRRI in Japanese female adolescents ( $n$ 63) aged 12-13 years (FFQW82, FFQ with eighty-two food items; 7d-FRRI, 7d weighed food record reported intake; FFQ1, first administration of FFQW82; FFQ2, second administration of FFQW82) 
WA, USA) was used to obtain the nutrient compositions for items on the FFQW82 ${ }^{(18)}$.

\section{7d-FRRI}

With the 7d-FRRI, actual intake was calculated based on weight of foods consumed. Specifically, the average intake for a whole day and for each meal was calculated separately. Food intake in the morning was included under the category of breakfast, that in the afternoon was considered as lunch, and that at night was under the category of dinner. These categories matched those in the FFQW82. All reports were reviewed by dietitians. After examining the completed 7d-FRRI, dietitians contacted the subjects to obtain missing or ambiguous information.

\section{Statistical methods}

Summary statistics were calculated. The data were logarithmically transformed to achieve normal distributions. In the case of no intake ( 0 value), we added 1 as a small value.

To assess the true validity of an FFQ would require measuring with high accuracy the usual self-selected diet of individuals corresponding to the duration of the FFQ. However, that is not feasible. Therefore, we assessed relative validity by comparing the FFQ with a 7d-FRRI as an alternative dietary assessment method; we assumed the latter as the 'gold standard.' The Pearson correlation coefficient between the reported intake from the 7d-FRRI and the estimated intake from the FFQ2 was calculated for each food group, for the whole day and for each meal, as well as for each of the nine nutrients shown in Table 2. We used the average of seven days for the 7d-FRRI as the reported intake when we calculated Pearson and Spearman correlation coefficients.

Reproducibility (test-retest reliability) was evaluated by the correlation between the estimated intake from results of the FFQ1 and the FFQ2. Crude and energy-adjusted Pearson correlation coefficients ${ }^{(9)}$ were calculated.

Because categories of intake, such as meals, snacks, food groups, etc., included no intake (0 values) and logarithmic transformations did not necessarily achieve normal distributions, the Spearman correlation coefficient was also calculated for both validity and reproducibility. In addition, due to the skewness of the nutrient data, we added a sensitivity analysis for transformation. Namely, the nutrient values were transformed to approximate normality using a Box-Cox transformation; thereafter, the Pearson correlation coefficient was calculated. The SAS for Windows statistical software package version 9.13 (SAS Institute, Cary, NC, USA) was used for the analysis.

\section{Results}

Table 1 shows basic statistics for estimated energy intake and actual energy intake by food group for a whole day and for each meal in sixty-three subjects. The median of the difference between estimated energy intake and actual energy intake was largest for lunch $(457 \mathrm{~kJ})$ and was $769 \mathrm{~kJ}$ for the whole day. Among food groups, the difference was particularly large for meat $(-183 \mathrm{~kJ})$ at dinner, but, for the whole day, the difference for sweets/ snacks ( $381 \mathrm{~kJ})$ was largest. As shown in Table 2, with regard to nutrients, the crude differences were greater for $\mathrm{K}(314 \mathrm{mg})$ and $\mathrm{Ca}(130 \mathrm{mg})$.

Results for validity of the FFQW82 by food group for a whole day and for meals are shown in Table 3. For daily total energy intake, the Pearson correlation coefficient was 0.31 and ranged from 0.18 (grains, fish) to 0.80 (eggs); the Spearman correlation coefficient ranged from $0 \cdot 12$ (grains) to 0.74 (milk). Table 4 shows results for validity with regard to nutrients. The energy-adjusted Pearson correlation coefficients for macronutrients ranged from $0 \cdot 28$ (carbohydrate) to 0.53 (protein). The Spearman correlation coefficients ranged from $0 \cdot 19$ (salt) to $0.55(\mathrm{Ca})$.

With regard to reproducibility, the Pearson correlation coefficient for total energy intake was 0.62 (energy) and for the food groups ranged from 0.11 (fat) to 0.83 (milk; Table 3). Spearman correlation coefficients ranged from 0.13 (fat) to 0.86 (milk; Table 3). As for the Box-Cox transformation, the results did not differ greatly from those of $\log$ transformation and varied from $0 \cdot 26(\mathrm{Fe})$ to 0.52 (Ca; results not shown).

\section{Discussion}

The FFQW82 has the advantage of assessing dietary intake at meals, which makes it possible to devise nutrition education to encourage young people to have adequate intake at breakfast. This is one of the important points for development of the FFQW82. Although not high, the Pearson correlation for total energy was about the same as in other studies (see Table 5) and did not differ greatly from the Spearman correlation.

\section{Characteristics of the FFQW82}

Several dietary instruments have been used to collect information on food intake to estimate the distribution of average intake of nutrients and foods in a population and to monitor such intake over time. Tooze et $\mathrm{ll}^{(20)}$ aimed to increase the precision of the predicted usual intake and of the estimated diet-health outcome relationships by combining two or more instruments. Our purpose is, as described above, to interpret correlations between the FFQW82 and 7d-FRRI, although we recognize that these instruments have limitations. It is important that an FFQ should be concise and easy to answer when assessing food intake ${ }^{(21)}$, especially in young people whose knowledge of food and cooking is generally limited. The FFQW82 was designed to present eighty-two food items according to food group and separately question intake frequency and portion size of each food at meals. The kind and quantity 
Table 1 Basic statistics on estimated energy (kJ) from FFQW82 and actual energy (kJ) from 7d-FRRI by food group (whole day, breakfast, lunch and dinner) in Japanese female adolescents $(n 63)$ aged $12-13$ years

\begin{tabular}{|c|c|c|c|c|c|c|c|c|c|c|c|c|}
\hline \multirow[b]{2}{*}{ Meal/Food group } & \multirow[b]{2}{*}{$n$} & \multicolumn{3}{|c|}{ Estimated energy } & \multirow{2}{*}{$\begin{array}{l}\% \text { with no } \\
\text { intake }\end{array}$} & \multicolumn{3}{|c|}{ Actual energy } & \multirow{2}{*}{$\begin{array}{l}\% \text { with no } \\
\text { intake }\end{array}$} & \multicolumn{3}{|c|}{ Difference } \\
\hline & & Median & P25 & P75 & & Median & P25 & P75 & & Median & P25 & P75 \\
\hline \multicolumn{13}{|l|}{ Whole day } \\
\hline Grains & 63 & 3170 & 2640 & 3814 & 0 & 2986 & 2746 & 3417 & 0 & 194 & -387 & 641 \\
\hline Fish & 63 & 238 & 165 & 481 & 0 & 256 & 152 & 377 & 3 & 48 & -105 & 176 \\
\hline Meat & 63 & 533 & 326 & 847 & 0 & 836 & 674 & 1084 & 2 & -306 & -493 & 16 \\
\hline Eggs & 63 & 142 & 41 & 319 & 5 & 215 & 120 & 299 & 5 & -22 & -97 & 49 \\
\hline Cheese & 63 & 30 & 0 & 90 & 25 & 41 & 0 & 88 & 38 & 0 & -24 & 29 \\
\hline Beans & 63 & 154 & 80 & 274 & 0 & 96 & 46 & 132 & 8 & 61 & -14 & 158 \\
\hline Vegetables & 63 & 226 & 131 & 335 & 0 & 176 & 128 & 266 & 0 & 27 & -51 & 114 \\
\hline Milk & 63 & 300 & 83 & 628 & 6 & 179 & 62 & 403 & 17 & 82 & -23 & 266 \\
\hline Fruits & 63 & 130 & 54 & 287 & 3 & 74 & 30 & 172 & 10 & 29 & -24 & 120 \\
\hline Sweets/snacks & 63 & 664 & 279 & 1130 & 0 & 100 & 0 & 373 & 27 & 381 & 151 & 870 \\
\hline Fat & 63 & 614 & 394 & 830 & 0 & 684 & 476 & 859 & 2 & 26 & -216 & 170 \\
\hline Total & 63 & 6809 & 5631 & 9442 & 0 & 6313 & 5330 & 7234 & 0 & 769 & -355 & 2320 \\
\hline \multicolumn{13}{|l|}{ Breakfast } \\
\hline Grains & 63 & 839 & 495 & 946 & 0 & 697 & 500 & 893 & 0 & 62 & -199 & 290 \\
\hline Fish & 63 & 9 & 0 & 69 & 35 & 0 & 0 & 16 & 62 & 1 & 0 & 41 \\
\hline Meat & 63 & 35 & 9 & 83 & 22 & 38 & 0 & 120 & 37 & 0 & -34 & 12 \\
\hline Eggs & 51 & 41 & 10 & 82 & 22 & 72 & 0 & 119 & 29 & 0 & -62 & 11 \\
\hline Cheese & 54 & 15 & 0 & 60 & 46 & 0 & 0 & 41 & 63 & 0 & 0 & 15 \\
\hline Beans & 63 & 12 & 0 & 56 & 41 & 0 & 0 & 16 & 63 & 0 & 0 & 28 \\
\hline Vegetables & 63 & 25 & 4 & 51 & 19 & 13 & 0 & 39 & 25 & 3 & 0 & 19 \\
\hline Milk & 63 & 209 & 23 & 345 & 14 & 158 & 2 & 282 & 24 & 0 & -42 & 88 \\
\hline Fruits & 60 & 20 & 0 & 88 & 30 & 20 & 0 & 84 & 45 & 0 & -20 & 22 \\
\hline Sweets/snacks & 63 & 106 & 21 & 333 & 21 & 0 & 0 & 88 & 63 & 32 & 0 & 162 \\
\hline Fat & 63 & 55 & 15 & 136 & 17 & 64 & 0 & 133 & 29 & 0 & -38 & 43 \\
\hline Total & 63 & 1602 & 1250 & 2320 & 0 & 1458 & 1090 & 1860 & 0 & 187 & -218 & 833 \\
\hline \multicolumn{13}{|l|}{ Lunch } \\
\hline Grains & 63 & 1223 & 972 & 1527 & 0 & 1150 & 909 & 1321 & 0 & 16 & -173 & 449 \\
\hline Fish & 49 & 64 & 28 & 125 & 0 & 33 & 10 & 63 & 16 & 23 & -16 & 90 \\
\hline Meat & 61 & 229 & 121 & 370 & 0 & 322 & 236 & 428 & 0 & -53 & -237 & 90 \\
\hline Eggs & 48 & 95 & 41 & 190 & 0 & 110 & 63 & 152 & 0 & 10 & -45 & 74 \\
\hline Cheese & 29 & 15 & 15 & 60 & 0 & 20 & 0 & 48 & 15 & 5 & -21 & 19 \\
\hline Beans & 39 & 27 & 14 & 75 & 0 & 6 & 0 & 23 & 25 & 17 & 6 & 64 \\
\hline Vegetables & 62 & 56 & 30 & 92 & 0 & 38 & 23 & 61 & 5 & 13 & -2 & 55 \\
\hline Milk & 23 & 46 & 11 & 102 & 0 & 0 & 0 & 7 & 24 & 22 & 11 & 102 \\
\hline Fruits & 46 & 43 & 17 & 87 & 0 & 19 & 0 & 62 & 26 & 22 & -3 & 49 \\
\hline Sweets/snacks & 62 & 232 & 128 & 499 & 0 & 37 & 0 & 167 & 46 & 158 & 62 & 346 \\
\hline Fat & 61 & 260 & 133 & 465 & 0 & 219 & 117 & 330 & 13 & 95 & -38 & 258 \\
\hline Total & 63 & 2385 & 1924 & 3305 & 0 & 2071 & 1652 & 2476 & 0 & 457 & 26 & 991 \\
\hline \multicolumn{13}{|l|}{ Dinner } \\
\hline Grains & 63 & 1191 & 1063 & 1419 & 0 & 1127 & 998 & 1373 & 0 & 31 & -255 & 257 \\
\hline Fish & 63 & 170 & 108 & 319 & 0 & 189 & 126 & 327 & 6 & -12 & -152 & 82 \\
\hline Meat & 63 & 237 & 147 & 486 & 2 & 456 & 321 & 611 & 2 & -183 & -353 & 0 \\
\hline Eggs & 56 & 20 & 20 & 82 & 0 & 76 & 29 & 97 & 2 & -9 & -60 & 16 \\
\hline Cheese & 60 & 15 & 0 & 15 & 40 & 0 & 0 & 20 & 68 & 0 & 0 & 15 \\
\hline Beans & 63 & 85 & 40 & 174 & 3 & 61 & 21 & 105 & 13 & 36 & -21 & 105 \\
\hline Vegetables & 63 & 131 & 78 & 188 & 0 & 130 & 77 & 180 & 0 & -4 & -59 & 57 \\
\hline Milk & 63 & 43 & 0 & 213 & 25 & 0 & 0 & 40 & 59 & 26 & 0 & 148 \\
\hline Fruits & 62 & 61 & 22 & 87 & 11 & 12 & 0 & 51 & 40 & 26 & 0 & 82 \\
\hline Sweets/snacks & 63 & 142 & 60 & 333 & 6 & 0 & 0 & 57 & 65 & 98 & 33 & 326 \\
\hline Fat & 63 & 220 & 107 & 376 & 0 & 329 & 241 & 474 & 2 & -85 & -232 & 50 \\
\hline Total & 63 & 2990 & 2234 & 3827 & 0 & 2719 & 2299 & 3239 & 0 & 198 & -353 & 683 \\
\hline
\end{tabular}

FFQW82, FFQ with eight-two food items; 7d-FRRI, 7d food record reported intake; estimated energy, estimated energy based on FFQW82; actual energy, actual energy from 7d-FRRI; difference, estimated energy minus actual energy; P25, 25th percentile; P75, 75th percentile.

of foods consumed usually differ among meals. Therefore, the method of asking about each meal individually in the FFQW82 allows for ease of use by the subjects. With this design, the FFQW82 makes it possible to estimate the energy intake by meals individually as well as by food group. The FFQW82 has the unique characteristic that it can be used as a tool for assessing the effect of nutritional education for each meal type ${ }^{(18)}$. In addition, the full-colour photographs might enable the subject to easily remember the actual foods eaten from the food list while doing the survey, make the survey easier to complete and increase the subject's motivation.

\section{7d-FRRI of female adolescents}

The validity of an FFQ is usually evaluated with food records, which are considered to be the 'gold standard' in 
Table 2 Basic statistics on estimated energy and actual energy for the whole day by nutrient in Japanese female adolescents ( $n$ 63) aged $12-13$ years

\begin{tabular}{|c|c|c|c|c|c|c|c|c|c|}
\hline \multirow[b]{2}{*}{ Nutrient } & \multicolumn{3}{|c|}{ Estimated energy } & \multicolumn{3}{|c|}{ Actual energy } & \multicolumn{3}{|c|}{ Difference } \\
\hline & Median & P25 & P75 & Median & P25 & P75 & Median & P25 & P75 \\
\hline Energy (kJ) & 6842 & 5631 & 9442 & 6313 & 5330 & 7234 & 769 & -355 & 2320 \\
\hline Protein $(\mathrm{g})$ & 58 & 47 & 86 & 56 & 46 & 67 & 5 & -8 & 25 \\
\hline Fat $(\mathrm{g})$ & 49 & 37 & 77 & 51 & 41 & 63 & 4 & -9 & 17 \\
\hline Carbohydrate (g) & 239 & 199 & 288 & 194 & 175 & 223 & 47 & 7 & 75 \\
\hline $\mathrm{Fe}(\mathrm{mg})$ & 6 & 5 & 8 & 5 & 4 & 6 & 0 & -1 & 3 \\
\hline $\mathrm{Ca}(\mathrm{mg})$ & 468 & 312 & 654 & 338 & 217 & 458 & 130 & 11 & 251 \\
\hline $\mathrm{Mg}(\mathrm{mg})$ & 204 & 153 & 281 & 168 & 132 & 192 & 37 & -5 & 102 \\
\hline $\mathrm{K}(\mathrm{mg})$ & 2099 & 1462 & 2942 & 1679 & 1344 & 2109 & 314 & -187 & 913 \\
\hline Dietary fibre $(\mathrm{g})$ & 11 & 8 & 15 & 9 & 7 & 11 & 2 & 0 & 6 \\
\hline Salt (g) & 9 & 5 & 8 & 7 & 5 & 8 & 2 & 0 & 4 \\
\hline
\end{tabular}

Estimated energy, estimated energy based on FFQW82; FFQW82, FFQ with eight-two food items; actual energy, actual energy from 7d-FRRI; 7d-FRRI, $7 \mathrm{~d}$ food record reported intake; difference, estimated energy minus actual energy; P25, 25th percentile; P75, 75th percentile.

Table 3 Validity and reproducibility of FFQW82 by food group in Japanese female adolescents $(n$ 63) aged $12-13$ years

\begin{tabular}{|c|c|c|c|c|c|c|c|c|c|c|}
\hline \multirow[b]{3}{*}{ Food group } & \multicolumn{8}{|c|}{ Validity* } & \multicolumn{2}{|c|}{ Reproducibility† } \\
\hline & \multicolumn{4}{|c|}{ Pearson } & \multicolumn{4}{|c|}{ Spearman } & \multirow{2}{*}{$\frac{\text { Pearson }}{\text { Whole day }}$} & \multirow{2}{*}{$\frac{\text { Spearman }}{\text { Whole day }}$} \\
\hline & Whole day & Breakfast & Lunch & Dinner & Whole day & Breakfast & Lunch & Dinner & & \\
\hline Grains & $0 \cdot 18$ & 0.32 & 0.40 & $0 \cdot 14$ & $0 \cdot 12$ & 0.20 & 0.25 & 0.05 & 0.54 & 0.63 \\
\hline Fish & $0 \cdot 18$ & 0.47 & $0 \cdot 14$ & 0.25 & 0.28 & 0.27 & $0 \cdot 15$ & $0 \cdot 16$ & 0.63 & 0.64 \\
\hline Meat & 0.59 & 0.72 & 0.36 & 0.55 & $0 \cdot 20$ & 0.51 & $0 \cdot 18$ & $0 \cdot 12$ & 0.54 & 0.46 \\
\hline Eggs & 0.80 & $0 \cdot 71$ & 0.42 & 0.22 & 0.58 & 0.53 & 0.39 & 0.34 & 0.60 & 0.43 \\
\hline Cheese & 0.71 & 0.69 & -0.35 & 0.47 & 0.44 & $0 \cdot 70$ & -0.24 & 0.57 & 0.69 & 0.53 \\
\hline Beans & 0.52 & 0.59 & 0.38 & 0.45 & 0.37 & 0.49 & 0.28 & $0 \cdot 31$ & 0.56 & 0.52 \\
\hline Vegetables & 0.46 & 0.79 & 0.47 & 0.41 & 0.32 & 0.50 & 0.34 & $0 \cdot 18$ & $0 \cdot 77$ & 0.66 \\
\hline Milk & 0.76 & $0 \cdot 81$ & 0.36 & 0.57 & $0 \cdot 74$ & $0 \cdot 76$ & $0 \cdot 18$ & 0.62 & 0.83 & $0 \cdot 86$ \\
\hline Fruits & 0.59 & 0.67 & $0 \cdot 15$ & 0.44 & 0.41 & 0.48 & -0.03 & 0.52 & 0.49 & 0.34 \\
\hline Sweets/snacks & 0.49 & 0.55 & $0 \cdot 40$ & 0.35 & 0.35 & 0.35 & 0.23 & $0 \cdot 29$ & 0.46 & 0.48 \\
\hline Fat & 0.67 & 0.73 & 0.39 & 0.54 & 0.41 & 0.50 & 0.37 & 0.35 & $0 \cdot 11$ & $0 \cdot 13$ \\
\hline Total & 0.31 & 0.59 & 0.40 & 0.32 & 0.28 & 0.49 & 0.37 & 0.25 & 0.62 & 0.58 \\
\hline
\end{tabular}

FFQW82, FFQ with eight-two food items; FFQ2, second administration of FFQW82; 7d-FRRI, $7 \mathrm{~d}$ food record reported intake; FFQ1, first administration of FFQW82.

${ }^{*}$ Correlation of estimated energy from FFQ2 and actual energy from the 7d-FRRI.

tCorrelation of the estimated energy from FFQ1 and FFQ2.

Table 4 Validity and reproducibility of FFQW82 by nutrient in Japanese female adolescents $(n$ 63) aged $12-13$ years

\begin{tabular}{|c|c|c|c|c|c|}
\hline \multirow[b]{2}{*}{ Nutrient } & \multicolumn{3}{|c|}{ Validity* } & \multicolumn{2}{|c|}{ Reproducibility† } \\
\hline & Pearson & Energy adjusted & Spearman & Pearson & Spearman \\
\hline Energy $(\mathrm{kJ})$ & 0.31 & - & 0.28 & 0.62 & 0.58 \\
\hline Protein $(\mathrm{g})$ & 0.35 & 0.53 & 0.33 & 0.62 & 0.56 \\
\hline Fat $(\mathrm{g})$ & 0.37 & 0.42 & 0.32 & 0.46 & 0.34 \\
\hline Carbohydrate $(\mathrm{g})$ & 0.29 & 0.28 & 0.25 & 0.69 & $0 \cdot 70$ \\
\hline $\mathrm{Fe}(\mathrm{mg})$ & 0.31 & 0.31 & 0.29 & 0.63 & 0.57 \\
\hline $\mathrm{Ca}(\mathrm{mg})$ & 0.52 & 0.49 & 0.55 & 0.76 & $0 \cdot 70$ \\
\hline $\mathrm{Mg}(\mathrm{mg})$ & $0 \cdot 37$ & $0 \cdot 42$ & 0.36 & $0 \cdot 70$ & 0.63 \\
\hline $\mathrm{K}(\mathrm{mg})$ & 0.37 & 0.53 & $0 \cdot 40$ & 0.73 & 0.65 \\
\hline Dietary fibre $(\mathrm{g})$ & 0.29 & 0.45 & 0.25 & 0.74 & 0.68 \\
\hline Salt (g) & 0.35 & 0.38 & $0 \cdot 19$ & 0.66 & 0.62 \\
\hline
\end{tabular}

FFQW82, FFQ with eight-two food items; FFQ2, second administration of FFQW82; 7d-FRRI, $7 \mathrm{~d}$ food record reported intake; FFQ1, first administration of FFQW82.

${ }^{*}$ Correlation of estimated energy from FFQ2 and actual energy from the 7d-FRRI.

tCorrelation of estimated energy from FFQ1 and FFQ2.

nutritional epidemiology ${ }^{(8)}$. Because the FFQW82 asks about intake over a period of 1 month, in making comparisons of its usefulness, it is desirable to keep food records for the same period of time. However, in studies of young people, we should consider their limited knowledge, skills and motivation for a dietary study ${ }^{(22)}$. 
Table 5 General information and adjusted correlation coefficients of validation studies of youth/adolescent FFQ

\begin{tabular}{|c|c|c|c|c|c|c|c|c|c|c|c|c|}
\hline$\overline{\text { Study }}$ & Our study & Rockett et al. ${ }^{(15)}$ & \multicolumn{2}{|c|}{ Slater et al. ${ }^{(14)}$} & Lietz et al. ${ }^{(13)}$ & Yaroch et al. ${ }^{(11)}$ & Maclntyre et al. ${ }^{(12)}$ & \multirow{2}{*}{\multicolumn{2}{|c|}{$\begin{array}{l}\text { Field et al.(10) } \\
1999\end{array}$}} & \multicolumn{3}{|c|}{ Rockett et al..$^{(9)}$} \\
\hline Publication year & 2010 & 2007 & \multicolumn{2}{|c|}{2003} & 2002 & 2000 & 2000 & & & \multicolumn{3}{|c|}{1997} \\
\hline Survey year(s) & 2007 & 1993-1994 & \multirow{2}{*}{\multicolumn{2}{|c|}{$\begin{array}{l}1999 \\
\text { Brazil }\end{array}$}} & $2000-2001$ & 1992 & $1996-1998$ & \multicolumn{2}{|l|}{$\begin{array}{l}\text { 1993-1994 } \\
\text { USA }\end{array}$} & \multicolumn{3}{|l|}{ 1993-1994 } \\
\hline Country & Japan & USA & & & UK & USA & South Africa & \multicolumn{2}{|l|}{ USA } & \multicolumn{3}{|l|}{ USA } \\
\hline $\begin{array}{l}\text { Reference data for } \\
\text { validation }\end{array}$ & $7 \mathrm{~d}$ diary record & $\begin{array}{l}3 \times 24 \mathrm{~h} \text { dietary } \\
\text { recalls }\end{array}$ & \multicolumn{2}{|c|}{$3 \times 24 \mathrm{~h}$ dietary recalls } & $\begin{array}{l}7 \mathrm{~d} \text { weighed dietary } \\
\text { record }\end{array}$ & $\begin{array}{l}3 \times 24 \mathrm{~h} \text { dietary } \\
\text { recalls }\end{array}$ & $\begin{array}{l}7 \mathrm{~d} \text { weighed dietary } \\
\text { record }\end{array}$ & \multicolumn{2}{|c|}{$\begin{array}{l}4 \times 24 \mathrm{~h} \text { dietary recalls over } \\
1 \text {-year period }\end{array}$} & \multicolumn{3}{|c|}{$3 \times 24 \mathrm{~h}$ dietary recalls } \\
\hline FFQ type & Self-administered & Self-administered & \multirow{2}{*}{\multicolumn{2}{|c|}{$\begin{array}{l}\text { Self-administered } \\
\text { Only once }\end{array}$}} & Interview-based & Picture-sort & Interview-based & \multirow{2}{*}{\multicolumn{2}{|c|}{ Semi-quantitative }} & \multicolumn{3}{|c|}{ Self-administered } \\
\hline FFQ interval & 1 month & 1 year & & & Only once & 2 weeks & $6-12$ weeks & & & \multirow{2}{*}{\multicolumn{3}{|c|}{$\begin{array}{l}1 \text { year } \\
126\end{array}$}} \\
\hline FFQ items & 82 & 26 & \multicolumn{2}{|c|}{76} & 131 & 97 & 145 & \multicolumn{2}{|l|}{97} & & & \\
\hline Sample size & 63 & 261 & 79 & & 50 & 22 & 74 & \multicolumn{2}{|l|}{109} & \multicolumn{3}{|l|}{261} \\
\hline Gender & All girls & $\begin{array}{l}\text { Girls } 53 \% \\
\quad \text { boys } 47 \%\end{array}$ & \multicolumn{2}{|c|}{$\begin{array}{l}\text { Girls } 49.4 \% \\
\quad \text { boys } 50.6 \%\end{array}$} & $\begin{array}{l}\text { Girls } 64 \% \\
\quad \text { boys } 36 \%\end{array}$ & All girls & $\begin{array}{l}\text { Women } 80 \% \\
\text { men } 20 \%\end{array}$ & \multicolumn{2}{|c|}{ Girls $52 \%$, boys $48 \%$} & \multicolumn{3}{|c|}{ Girls $53 \%$, boys $47 \%$} \\
\hline Age (years) & $13-14$ & $9-18$ & $14-18$ & & $11-13$ & $11-17^{*}$ & $15-65$ & \multirow{2}{*}{\multicolumn{2}{|c|}{$\begin{array}{l}\text { 4-7th gradet } \\
\text { Spearman }\end{array}$}} & \multirow{2}{*}{\multicolumn{3}{|c|}{$\begin{array}{l}9-18 \\
\text { Pearson }\end{array}$}} \\
\hline $\begin{array}{l}\text { Correlation } \\
\text { coefficient }\end{array}$ & Pearson & Pearson & Pearson & & Spearman & Pearson & Spearman rank & & & & & \\
\hline Nutrient & Total & Total & Female & Male & Total & Total & Total & $4-5$ th grade & $6-7$ th grade & $9-13$ years & $14-18$ years & Total \\
\hline Energy (kcal) & 0.31 & 0.34 & 0.86 & 0.82 & 0.33 & 0.63 & 0.31 & 0.26 & 0.47 & 0.21 & 0.49 & 0.35 \\
\hline Protein $(\mathrm{g})$ & 0.53 & 0.30 & $0 \cdot 17$ & $0 \cdot 21$ & 0.31 & 0.32 & 0.30 & 0.21 & 0.43 & 0.28 & 0.47 & 0.37 \\
\hline Fat $(\mathrm{g})$ & 0.42 & 0.41 & -0.07 & 0.33 & 0.66 & 0.59 & 0.25 & 0.24 & 0.44 & 0.44 & 0.54 & 0.49 \\
\hline Carbohydrate (g) & 0.28 & 0.33 & 0.12 & 0.43 & 0.50 & 0.65 & 0.31 & 0.22 & 0.47 & 0.42 & 0.39 & 0.40 \\
\hline $\mathrm{K}(\mathrm{mg})$ & 0.53 & 0.32 & - & - & 0.60 & - & - & - & - & 0.41 & 0.48 & 0.44 \\
\hline $\mathrm{Ca}(\mathrm{mg})$ & 0.53 & 0.54 & 0.50 & 0.46 & 0.47 & - & 0.24 & 0.35 & 0.55 & 0.52 & 0.57 & 0.55 \\
\hline $\mathrm{Mg}(\mathrm{mg})$ & 0.37 & 0.45 & - & - & - & - & - & - & - & 0.51 & 0.54 & 0.52 \\
\hline $\mathrm{Fe}(\mathrm{mg})$ & 0.31 & 0.51 & $0 \cdot 10$ & $0 \cdot 11$ & - & - & 0.20 & 0.04 & 0.35 & 0.47 & 0.59 & 0.53 \\
\hline Dietary fibre (g) & 0.45 & 0.41 & 0.48 & 0.62 & $0.49 \ddagger$ & - & 0.14 & 0.06 & 0.45 & 0.46 & 0.46 & 0.46 \\
\hline $\mathrm{Na}(\mathrm{mg})$ & - & 0.05 & - & - & 0.26 & - & - & - & - & 0.13 & 0.32 & 0.21 \\
\hline Salt (g) & 0.38 & - & - & - & - & - & - & - & - & - & - & - \\
\hline
\end{tabular}

*Subjects were all low-income, overweight African-Americans.

tSubjects were all low-income and $84 \%$ were African-Americans.

‡Englyst fibre. 
Considering the feasibility of conducting a dietary survey, we used the average intake for seven continuous days (1 week) instead of the average intake over seven randomly selected days over a period of 1 month as an approximation. However, nearly half of the subjects did not complete the required $7 \mathrm{~d}$ of record keeping, with most not continuing to weigh food for the requested time. This is the limitation of our survey. The 'fatigue factor' might affect the quality of what is reported after 3 to $4 \mathrm{~d}$ in a $7 \mathrm{~d}$ dietary record, as the subject tires of the task and becomes negligent regarding details. Although we examined daily distribution of energy intake as well as daily fat intake, we did not find evidence of decreased quality in the reporting after 3 to $4 \mathrm{~d}$. The subjects who completed the survey did not differ from those who did as to age or instructions on use of the instrument. We felt that the data provided by the sixty-three remaining female subjects met our needs. Considering the lifestyle of adolescents, it is a potentially acceptable method to assess intake over a period of 1 week by weighed food records, including both a weekend and weekdays.

\section{Validity and reliability}

Rockett et al. ${ }^{(9)}$ conducted a study of the validity of their instrument in 149 subjects (9-13 years old) who were similar in age to our subjects. In their study, the correlation coefficient between the $24 \mathrm{~h}$ recall method for $3 \mathrm{~d}$ and the FFQ (126 items) was $0 \cdot 21$ for energy while that in our study was $0 \cdot 31$. Also in comparison with their results, our study found similar energy-adjusted correlation coefficients for macronutrients. With the exception of results by Slater et $a l^{(14)}$, which showed higher correlations for energy $(0 \cdot 86$ for females), the correlations were not very high (Table 5).

Divergence for sweets/snacks was shown in the present study, with an estimated intake of $664 \mathrm{~kJ}$ while the actual intake was $100 \mathrm{~kJ}$ for sweets/snacks, which were included in a list of confectioneries and beverages, and the median of the difference was $381 \mathrm{~kJ}$. This appeared to strongly influence the correlation for energy at $0 \cdot 31$. On the other hand, reproducibility for energy was high at $0 \cdot 62$. Therefore, it was thought that there was an omission at the time of recording the weight of sweets/snacks, including confectioneries and beverages, when completing the 7d-FRRI. In the study by Watson et al. ${ }^{(23)}$ participants who had not completed food records instead completed a $24 \mathrm{~h}$ recall of food intake. To use two or more instruments is a good way to improve the reference standard.

Marchioni et $a l^{(24)}$ studied the reproducibility of an FFQ consisting of seventy-six items with forty-six subjects aged 16-19 years. The correlation coefficient for energy was 0.53 for their study and 0.62 for our study. The energy-adjusted correlation coefficients were also very much higher in our study than in their study except for fat (ours $=0 \cdot 11$ ). This may be because under the category of 'fat' were foods such as seeds and oils, which are not easy to quantify on the FFQW82. Another reason is that half of the foods in the 'fat' group were mixtures of various constituents. Results might be improved if there was a direct question about the quantity of oils.

\section{Advantages and limitations of the study}

The present study has several advantages. First, to our knowledge, this is the first study to examine the validity between FFQ and 7d-FRRI in Japanese female adolescents. The advantage of the FFQW82 is that a habitual diet can be analysed by meal type (breakfast, lunch and dinner) and by food group. Second, specific problems related to dietary intake of young people, such as skipping breakfast or excessive intake of snacks at night, can be revealed and the goals of dietary improvement based on scientific evidence can be planned and practised. Especially, breakfast habits affect overall nutrient profiles ${ }^{(25,26)}$ and to ascertain the breakfast habits of this age group is important. Additionally, assessment of the effect of nutritional education may also be possible. Third, the validity for breakfast was higher than for lunch or dinner, which ranged from $0 \cdot 32$ (grains) to $0 \cdot 81$ (milk). Assessing problems by meal type is important for giving dietary guidance not only in Japan, but also overseas. The FFQW82 is designed to ask for the frequency of intake and the amount of a typical portion of each food for breakfast, lunch and dinner separately.

The study has some limitations. First, the subjects were limited to female adolescents $12-13$ years of age. Thus, we cannot discuss or draw conclusions on the dietary habits of male adolescents. As for the gender effect on the validity of the study, Rockett et $a l .{ }^{(15)}$ evaluated the validity of an FFQ in youths from 9 to 18 years old by gender. The results showed that the correlation coefficient for energy was 0.30 in males and 0.33 in females and for macronutrients ranged from 0.38 (protein) to 0.52 (fat) in males and from 0.35 (carbohydrate) to 0.49 (fat) in females. They indicated no gender difference in the validity of the FFQ with subjects in this age group. It appears that since the dietary needs at these ages are fulfilled primarily at home, the food content is almost the same but the amount of intake differs. Therefore, they suggested that no gender difference existed in the correlations. The FFQW82 might be used for male adolescents; however, there is the need for some additional work in the future if young males are to be served by this instrument. Second, data for only sixty-three of the 121 students were used in the final analysis. The remaining subjects either did not complete at least six of the seven days of the dietary record or missed recording several meals. As for possible factors that may be associated with use of the FFQW82 and 7d-FRRI between subjects who did and did not complete the 7d-FRRI, we examined differences among subjects who did not and who did complete the dietary record in terms of BMI, sports 
activity, dietary behaviour, hours of sleep and hours of study at home, from information obtained previously through a self-administered questionnaire (results not shown here). However, there was no significant difference $(P>0 \cdot 05)$ between the two groups for BMI (19.1 $v$. $\left.18.6 \mathrm{~kg} / \mathrm{m}^{2}\right)$, sports activities and other responses. From these potential factors, we interpreted that the effects of bias might be not so large, although we cannot prove it. Because the aim of the study was to assess the correlations between FFQW82 and 7d-FRRI, this may not seriously bias the results. Third, the fact that the estimated correlations between the FFQ and 7d-FRRI were 'relatively high' does not generally mean that the FFQ is 'acceptable to use' when both have correlation errors. However, the 7d-FRRI is more objective and the correlation bias of self-report might be less with this instrument. Furthermore, the 7d-FRRI, which involved the use of photographs, was reviewed by dietitians. Thus, this may reduce bias. It might be possible that the subjects underate during the recording period. Although the actual intake was lower than the estimated intake, this does not necessarily indicate that these subjects under-ate for the entire week, with the exception of sweets/snacks. That is, both the median of the difference and the percentage of no intake in sweets/snacks were large and the chance of reporting under-eating was high. This should be interpreted carefully. Fourth, FFQ are generally recognized to involve substantial measurement error, both random and systematic. In the present study, because of limitation of the data, we cannot examine this point. As for the Spearman correlation coefficient, it can be used for ordinal or categorical data even when there is a spike at zero in the data and it may be helpful in interpretation of the association.

\section{Conclusions}

In the current study, the validity and reproducibility of the FFQW82 in female adolescents were evaluated. This instrument, originally developed for use in adults, has proved to have some potential with regard to reproducibility among our study population. However, the study only showed reliability for intake at breakfast, as reliability was somewhat lower for lunch and dinner in female adolescents. Further work is necessary to refine this instrument to make it more valid for lunch and dinner.

\section{Acknowledgements}

The study was financially supported by the Ministry of Education, Culture, Sports, Science and Technology in Japan Grant-in-Aid for Scientific Research Grant C in 2005-2007 (Grant No. 17500551) and 2008 (Grant No. 19500693). The corresponding author confirms all coauthors have no conflict of interest to declare. There have been no involvements that might raise the question of bias in the work reported or in the conclusions, implications or opinions stated. All financial research or project support has been clearly stated. Author contributions are as follows: M.W., K.Y. and T.T. decided the study design; M.W., M.Y. and M.A. conducted the dietary survey and calculated nutritional intakes; K.Y. and T.T. did data analysis. All authors contributed to write the paper and discussed the results.

\section{References}

1. Freedman DS, Kahn HS, Mei Z et al. (2007) Relation of body mass index and waist-to-height ratio to cardiovascular disease risk factors in children and adolescents: the Bogalusa Heart Study. Am J Clin Nutr 86, 33-40.

2. Japan Ministry of Education, Culture, Sports, Science and Technology (2006) Annual Report of School Health Statistics Research. Tokyo: National Printing Bureau.

3. Van Dam RM, Willett WC, Manson JE et al. (2006) The relationship between overweight in adolescence and premature death in women. Ann Intern Med 145, 91-97.

4. Strong JP, Malcom GT, McMahan AC et al. (1999) Prevalence and extent of atherosclerosis in adolescents and young adults: implications for prevention from the PDY study. JAMA 281, 727-735.

5. Centers for Disease Control and Prevention (1997) Update: prevalence of overweight among children, adolescents, and adults - United States, 1988-1994. MMWR Morb Mortal Wkly Rep 46, 199-202.

6. Health and Nutrition Information Association (2006) 2004 Year National Health and Nutrition Report of Japan Ministry of Health, Labour and Welfare. Tokyo: Daiichi Syuppan.

7. Rennie KL, Jebb SA, Wright A et al. (2005) Secular trends in under-reporting in young people. Br J Nutr $\mathbf{9 3}$, 241-247.

8. Willett WC (1990) Nutritional Epidemiology. New York: Oxford University Press.

9. Rockett HRH, Breitenbach M, Frazier AL et al. (1997) Validation of a youth/adolescent food frequency questionnaire. Prev Med 26, 808-816.

10. Field AE, Peterson KE, Gortmaker SL et al. (1999) Reproducibility and validity of a food frequency questionnaire among fourth to seventh grade inner-city school children: implications of age and day-to-day variation in dietary intake. Public Health Nutr 2, 293-300.

11. Yaroch AL, Resnicow K, Davis M et al. (2000) Development of a modified picture-sort food frequency questionnaire administered to low-income, overweight, African-American adolescent girls. J Am Diet Assoc 100, 1050-1056.

12. Maclntyre UE, Venter CS \& Vorster HH (2000) A culturesensitive quantitative food frequency questionnaire used in an African population: 2. Relative validation by 7-day weighted records and biomarkers. Public Health Nutr 4, 63-71.

13. Lietz G, Barton KL, Longbottom PJ et al. (2002) Can the EPIC food-frequency questionnaire be used in adolescent populations? Public Health Nutr 5, 783-789.

14. Slater B, Philippi ST, Fisberg RM et al. (2003) Validation of semi-quantitative adolescent food frequency questionnaire applied at a public school in Sao Paulo, Brazil. Eur J Clin Nutr 57, 629-635.

15. Rockett HRH, Berkey CS \& Colditz GA (2007) Comparison of a short food frequency questionnaire with the Youth/ Adolescent Questionnaire in the Growing Up Today study. Int J Pediatr Obes 2, 31-39. 
16. Matthys C, Pynaert I, De Keyzer W et al. (2007) Validity and reproducibility of an adolescent web-based food frequency questionnaire. J Am Diet Assoc 107, 605-610.

17. Yamaoka K, Tango T, Watanabe M et al. (2000) Validity and reproducibility of a semi-quantitative food frequency questionnaire for nutritional education of patients of diabetes mellitus (FFQW65). Jpn J Public Health 47, 230-244.

18. Adachi M, Watanabe M, Yamaoka K et al. (2010) Validity and reproducibility of food frequency questionnaire with 82-food lists (FFQW82) for nutrition education. Jpn J Public Health 57 (In the Press).

19. Watanabe M, Yamaoka K, Yokotsuka M et al. (2003) Randomized controlled trial of a new dietary education program to prevent type 2 diabetes in a high-risk group of Japanese male workers. Diabetes Care 26, 3209-3214.

20. Tooze JA, Midthune D, Dodd KW et al. (2006) A new statistical method for estimating the usual intake of episodically consumed foods with application to their distribution. J Am Diet Assoc 106, 1575-1587.
21. Beaton GH, Milner J, Corey P et al. (1979) Sources of variance in 24-hour dietary recall data: implications for nutrition study design and interpretation. Eur J Clin Nutr 32, 2546-2559.

22. Frank GC, Berenson GS, Schilling PE et al. (1977) Adapting the 24-hr recall for epidemiologic studies of school children. J Am Diet Assoc 71, 26-31.

23. Watson JF, Collins CE, Sibbritt DW et al. (2009) Reproducibility and comparative validity of a food frequency questionnaire for Australian children and adolescents. Int J Behav Nutr Phys Act 6, 62.

24. Marchioni DML, Voci SM, de Lima FEL et al. (2007) Reproducibility of a food frequency questionnaire for adolescents. Cad Saude Publica 23, 2187-2196.

25. Matthys C, De Henauw S, Bellemans M et al. (2007) Breakfast habits affect overall nutrient profiles in adolescents. Public Health Nutr 10, 413-421.

26. Chitra U \& Reddy CR (2007) The role of breakfast in nutrient intake of urban schoolchildren. Public Health Nutr 10, 55-58. 